\title{
Uso de serviços odontológicos de forma regular na população de Montes Claros, MG, Brasil
}

\author{
The use of dental services on a regular basis in the population \\ of Montes Claros in the State of Minas Gerais, Brazil
}

Danilo Lima Carreiro ${ }^{1}$

João Gabriel Silva Souza ${ }^{2}$

Wagner Luiz Mineiro Coutinho ${ }^{3}$

Raquel Conceição Ferreira ${ }^{4}$

Efigênia Ferreira e Ferreira ${ }^{4}$

Andrea Maria Eleutério de Barros Lima Martins ${ }^{5}$

${ }^{1}$ Instituto Federal de

Educação, Ciência e

Tecnologia do Norte de

Minas. R. Cel. Luís Píres

202, Centro. 39400-106

Montes Claros MG Brasil.

danilolimacarreiro@

gmail.com

${ }^{2}$ Faculdade de Odontologia de Piracicaba, Universidade

Estadual de Campinas.

Piracicaba SP Brasil.

${ }^{3}$ Instituto de Ciências da

Saúde, Associação Educativa

do Brasil. Montes Claros

MG Brasil.

${ }^{4}$ Faculdade de Odontologia,

Universidade Federal

de Minas Gerais. Belo

Horizonte MG Brasil.

${ }^{5}$ Departamento de

Odontologia, Universidade

Estadual de Montes Claros.

Montes Claros MG Brasil.
Abstract The use of dental services on a regular basis by residents of Montes Claros (MG) was assessed, as well as related aspects. Cross-sectional study was conducted with a complex probabilistic sample by conglomerates. Logistic regression was used (OR/CI95\%) with a significance level of 5. Of the 2582 study participants, 761 required and obtained access to the services for less than a year. Of these, 262 (35.8\%) used the services in a regular/routine manner. The use of dental services on a regular basis was greatest among individuals in the age group " $\geq 18 \leq 37$ "; with schooling "equal to or greater than 9 years"; who classified their oral health, general health and appearance of teeth and gums as "excellent/good"; who reported no pain" on teeth and gums over the last six months; They reported that the "assistance provider had adequate skills"; that they "always/often" received information about oral hygiene and diet/nutrition. In addition, this use was lower among those who rated the time to ask questions about the dental problem or treatment as "regular/bad/terrible." The conclusion drawn is that the use of dental services on a regular basis was relatively low, being associated with personal traits, subjective health conditions and characteristics related to the services.

Key words Preventive dentistry, Oral health, Dental health services
Resumo Avaliou-se o uso de serviços odontológicos de forma regular entre os residentes de Montes Claros (MG), assim como os aspectos relacionados a esse uso. Estudo transversal realizado com amostra complexa probabilística por conglomerados. Utilizou-se a análise de regressão logística (OR/IC95\%). Dos 2582 participantes do estudo, 761 necessitaram de serviços odontológicos e obtiveram acesso a eles há menos de um ano. Destes, 262 (35,8\%) fizeram uso dos serviços de forma regular. Esse uso foi maior entre os indivíduos na faixa etária " $\geq 18 \leq 37$ "; com escolaridade "igual ou maior a 9 anos"; que classificaram sua saúde bucal, saúde geral e a aparência dos dentes e gengivas como "ótima/boa"; que não relataram dor nos dentes e gengivas nos últimos seis meses; que relataram que o "prestador da assistência possuía habilidades adequadas"; que "sempre/frequentemente" receberam informações sobre higiene bucal e sobre dieta/alimentação. Em adição, esse uso foi menor entre os que classificaram o tempo para fazer perguntas sobre o problema bucal ou tratamento como "regular/ruim/péssimo". Conclui-se que o uso de serviços odontológicos de forma regular foi relativamente baixo, sendo associado a características pessoais, condições subjetivas de saúde e características relacionadas aos serviços.

Palavras-chave Odontologia preventiva, Saúde bucal, Serviços de saúde bucal 


\section{Introdução}

A atenção à saúde bucal no Brasil tem passado por transformações da assistência com um maior enfoque na promoção de saúde e prevenção de doenças, pautadas, principalmente na educação em saúde, requalificação da atenção e a ampliação do acesso aos serviços ${ }^{1-3}$. Apesar dos avanços alcançados no processo de atenção à saúde bucal e nas políticas públicas relacionados à esta atenção ${ }^{3,4}$, ainda pode-se constatar desigualdades sociais no uso de serviços odontológicos entre as regiões do Brasil e também entre os indivíduos ${ }^{5-7}$. Tal disparidade pode estar relacionada às características contextuais de cada realidade, bem como com as características individuais das pessoas. Além disso, observa-se uma alta prevalência de agravos bucais na população brasileira, tais como cárie dentária, doença periodontal e perdas dentárias, como demonstrado pelo último levantamento epidemiológico das condições de saúde bucal da população brasileira, realizado pelo Ministério da saúde em 2010 (SB Brasil 2010) ${ }^{8}$. Tal fato é ainda agravado quando se observa uma prevalência relevante de indivíduos que nunca fizeram uso de serviços odontológicos entre os diferentes contingentes populacionais ${ }^{8}$. Essa realidade em que o uso de serviços odontológicos é baixo demonstra a necessidade de consolidação das políticas públicas existentes de saúde que fomentem programas educativos, acesso da população e uso de serviços odontológicos de forma regular6.

Na literatura, verifica-se uma escassez de estudos que avaliaram o uso para a prevenção ou comportamentos odontológicos preventivos ${ }^{9}$. Ademais, os fatores associados aos diversos usos (preventivos, curativos ou reabilitadores) são distintos ${ }^{10}$. Cabe ressaltar que o uso regular é um hábito importante para a saúde bucal do sujeito ${ }^{6}$. O uso regular dos serviços pode ser um importante preditor da saúde bucal, podendo levar à execução de tratamentos de caráter preventivo ${ }^{11}$, evitando, assim, a ocorrência de problemas bucais e, consequentemente, reduzindo a propensão a perdas de horas de trabalho, decorrentes desses problemas ${ }^{12}$. Diferentes contingentes populacionais têm sido avaliados quanto ao uso regular de serviços odontológicos, tais como: pré-escolares $^{13}$, adultos ${ }^{14}$ e idosos ${ }^{6}$, sendo esse uso associado, principalmente, a melhores condições socioeconômicas, características do serviço e condições subjetivas de saúde $e^{6,13,14}$.

O modelo teórico mais utilizado, que tem como objetivo avaliar os fatores associados ao uso de serviços odontológicos, foi proposto por Andersen e Davidson ${ }^{10}$. Tal modelo foi desenvolvido para adultos e idosos nos Estados Unidos e demonstra que as variáveis exógenas (grupos étnicos e corte etário) possuem influência sobre os determinantes primários de saúde bucal (características do ambiente externo, saúde geral relatada, sistema de atenção à saúde bucal e características pessoais dos sujeitos). Estes últimos também possuem influência sobre comportamento de saúde bucal (compreendem as práticas pessoais e o uso formal de serviços, seja ele preventivo, curativo ou reabilitador) que, por sua vez, influenciam os desfechos em saúde bucal (condição de saúde bucal avaliada por um profissional da odontologia, percepção da condição de saúde bucal e a satisfação do paciente). Tal modelo tem sido utilizado com o intuito de identificar possíveis fatores associados ao uso de serviços odontológicos ${ }^{15}$.

Nesse sentido, verifica-se que os comportamentos de saúde bucal são variáveis intermediárias, possivelmente preditoras dos desfechos de saúde bucal e dependentes dos determinantes de saúde bucal. O uso de serviços odontológicos de forma regular é perceptível no domínio Comportamentos de Saúde Bucal. Tais comportamentos demonstram que o indivíduo faz o uso formal dos serviços, neste caso, para a prevenção; destaca-se que o modelo proposto por Andersen \& Davidson também considera as possibilidades de uso desses serviços com fins curativos ou reabilitadores. As interações apresentadas no modelo são dinâmicas e possuem caráter retroalimentador, em que os desfechos de saúde bucal determinam e são determinados pelos comportamentos de saúde bucal ${ }^{10}$.

No Brasil, constata-se uma carência de pesquisas que tenham investigado fatores associados ao uso de serviços odontológicos de forma regular. Portanto, o presente estudo teve por objetivo avaliar o uso dos serviços odontológicos de forma regular entre residentes de um município brasileiro de grande porte populacional. Considerou-se o modelo teórico proposto por Andersen e Davidson ${ }^{10}$.

\section{Metodologia}

Trata-se de um estudo transversal, realizado em 2012, na área urbana de Montes Claros (MG), sendo considerado um participante para cada unidade domiciliar, com idade $\geq 18$ anos e que tivesse utilizado serviços odontológicos nos últi- 
mos 12 meses. No cálculo amostral para a população finita (N=344.427/IBGE, 2010), considerouse a estimativa para proporções da ocorrência dos eventos ou doenças em $50 \%$ da população, um erro de $5 \%$, taxa de não resposta de $20 \%$, e um deff (design effect - efeito de desenho) de 2,0. No primeiro estágio do planejamento amostral por conglomerado, foram sorteados 30 setores censitários do município a partir da amostragem proporcional ao tamanho. No segundo estágio, sorteou-se um percentual de quadras em cada um dos 30 setores selecionados. Todos os domicílios das quadras selecionadas foram visitados e as pessoas que atendessem aos critérios de inclusão foram convidadas a participar do inquérito. Por fim, foram atribuídos pesos diferenciados aos elementos da amostra, sendo estes calculados através do inverso do produto das probabilidades de inclusão nos diversos estágios de seleção ${ }^{16}$. Estimou-se uma amostra de 2582 representantes domiciliares.

As entrevistas foram realizadas por estudantes dos cursos de graduação em medicina, odontologia e matemática, de uma instituição de ensino superior pública, que foram devidamente treinados.

A variável dependente - uso de serviços odontológicos de forma regular - foi definida entre aqueles que relataram ter utilizado o serviço no último ano e de forma regular. Para construção da variável dependente foram considerados os indivíduos que precisaram de assistência odontológica a menos de um ano e a obtiveram. A variável dependente foi construída a partir do relato de uso de serviços odontológicos de forma regular, identificado em duas questões, sendo elas: "Você vai ao dentista de forma regular ou periódica?" (sim e não); e "Que problema, melhor descreve por que você precisou de assistência odontológica?" (revisão/prevenção/uso regular/limpeza; sangramento na gengiva; dor de dente; dor na gengiva; colocar aparelho ortodôntico; necessidade de prótese; dor muscular ou próxima ao ouvido; fazer canal; necessidade de fazer restaurações; extrair dente; clarear dentes; outro). O relato de uso de forma regular em ambas as variáveis foi considerado para criação da dependente - essa variável foi nomeada uso de serviços odontológicos de forma regular e dicotomizada em "sim" e "não".

As variáveis independentes foram agrupadas a partir do modelo teórico de Andersen e Davidson $^{10}$, conforme descrito anteriormente ${ }^{15}$ : características pessoais; sistema de atenção à saúde bucal; autopercepção da saúde bucal; saúde geral re- latada; práticas pessoais e satisfação do paciente.

$\mathrm{O}$ grupo referente às características pessoais foi subdivido em predisposição e disponibilidade de recursos. Não foi construído um grupo de variáveis exógenas (estrato etário e etnia), pois, no modelo teórico, verificou-se apenas um único estrato etário e a etnia é apresentada conforme organização sociocultural que se difere do Brasil, em que se usa a terminologia raça/cor ${ }^{6}$. Nesse sentido, utilizou-se classificação de modo apropriado à realidade nacional e conforme o Instituto Brasileiro de Geografia e Estatística (IBGE). Portanto, compôs o subgrupo predisposição as variáveis: sexo; faixas etárias em anos, dicotomizadas pelo limite inferior do intervalo de confiança de 95\%; raça/cor declarada; escolaridade expressa em anos ${ }^{17}$ e estado civil. O subgrupo disponibilidade de recursos compreendeu: renda per capita. Avaliou-se também a situação ocupacional do respondente. Também foram avaliados nesse subgrupo as variáveis: meio de transporte utilizado para chegar ao local da consulta e tempo gasto para chegar ao local da consulta, categorizados pelos quartis.

Para avaliar o grupo referente ao sistema de atenção à saúde bucal, utilizaram-se as seguintes variáveis: cadastrado do domicílio no Programa de Saúde da Família; local da consulta; prestador da assistência odontológica; e como o serviço foi pago.

Compuseram o grupo da autopercepção da saúde bucal variáveis referentes à autopercepção da: saúde bucal geral; mastigação, aparência de dentes e gengivas; fala; relacionamento com outras pessoas decorrente das condições de saúde bucal; e dor de dentes e gengivas, nos últimos 6 meses.

A variável que compôs o grupo saúde geral relatada foi elaborada a partir da pergunta "como você classifica a sua saúde geral?” No grupo de variáveis relacionadas a práticas pessoais, avaliou-se o hábito de fazer o autoexame da boca.

$\mathrm{O}$ último grupo está relacionado à satisfação do paciente. Para tal, construiu-se um instrumento estruturado, baseado no questionário sobre avaliação da assistência oftalmológica -Questionário de Responsividade ao Sistema de Assistência Ocular (QRSAO) ${ }^{18}$. Esse instrumento aborda aspectos sobre a assistência ocular e foi desenvolvido pela Organização Mundial de Saúde (OMS) e traduzido para língua portuguesa e cultura brasileira ${ }^{18}$. É constituído por questões que abordam prontidão no atendimento, dignidade, privacidade, comunicação, autonomia, escolha e infraestrutura ${ }^{18}$. Optou-se pela modi- 
ficação desse instrumento por tratar-se de uma avaliação da responsividade do usuário quanto ao serviço utilizado, seguindo os parâmetros propostos pela OMS. Além disso, apesar de ter sido desenvolvido para a assistência oftalmológica, o instrumento não possui questões específicas apenas para esse tipo de serviço, sendo passível de serem modificadas. A confiabilidade desse instrumento (em sua versão para a assistência odontológica) foi avaliada (concordância boa) antes da coleta de dados e utilizado/descrito em estudo prévio ${ }^{19}$.

Além disso, o grupo satisfação do paciente considerou as seguintes variáveis: satisfação com os serviços odontológicos; se o prestador de assistência odontológica possuía habilidades adequadas; se os materiais e equipamentos do prestador de assistência odontológica eram adequados para o tratamento.

Para a análise dos dados, empregou-se o software SPSS ${ }^{\circledR}$ 19.0. Para avaliação dos fatores associados ao desfecho (uso regular), foram realizadas análises bivariada e múltipla. $\mathrm{Na}$ análise bivariada, foram estimadas odds ratio brutas (OR) e intervalos de confiança de 95\% ( $\mathrm{IC}_{95 \%}$ ), com a correção pelo efeito do desenho amostral. As variáveis independentes que apresentaram nível descritivo menor ou igual a 20\% ( $\mathrm{p} \leq 0,20)$ foram para a análise múltipla. Foram estimadas as magnitudes das associações do uso regular dos serviços odontológicos com as variáveis independentes investigadas. Utilizou-se regressão logística com correção pelo efeito de desenho, calculouse a Odds Ratio com nível de significância de 5\% e intervalos de confiança de 95\% (OR/IC 95\%).

A investigação atendeu aos princípios da Resolução 196/96 do Conselho Nacional de Saúde (CNS), tendo sido aprovada com parecer consubstanciado quanto à sua realização, pelo Comitê de Ética e Pesquisa da Associação Educativa do Brasil (SOEBRAS).

\section{Resultados}

Dos 2582 participantes do estudo, 2581 necessitaram de assistência odontológica. Destes, 1685 obtiveram $(63,8 \%)$. Dentre os que obtiveram a assistência, 791 (47,0\%) haviam sido atendidos há menos de um ano, e 29 não souberam informar. Sendo assim, o uso regular foi avaliado em 761 indivíduos, destes, 262 (35,8\%) relataram uso de forma regular (Figura 1).

Dentre os indivíduos incluídos, identificouse uma maior predominância de indivíduos com idade entre 18 e 37 anos, do sexo feminino, com renda per capita menor que um salário mínimo, e que haviam utilizado serviço odontológico particular/convênio (Tabela 1).

$\mathrm{Na}$ análise bivariada, identificou-se associação entre uso de serviços odontológicos de forma regular com variáveis referentes às características pessoais, sistema de atenção à saúde bucal, autopercepção da saúde bucal, saúde geral relatada, práticas pessoais, satisfação do paciente e domínios do QRSAO (Tabela 2).

$\mathrm{Na}$ Tabela 3, são apresentados os resultados do modelo final ajustado $(p \leq 0,05)$ com as variáveis que permaneceram significativamente associadas ao uso de serviços odontológicos de forma regular, sendo tais variáveis associadas: faixa etária, escolaridade, autopercepção da saúde bucal, aparência de dentes e gengivas, dor de dentes, autopercepção da saúde geral, habilidades do profissional na percepção do paciente e variáveis do domínio da comunicação.

\section{Discussão}

Ao considerar a adaptação ao modelo teórico de Andersen e Davidson, utilizado no presente estudo, identificou-se que o uso de serviços odontológicos de forma regular manteve-se associado a grupos de variáveis preconizadas pelo modelo (características pessoais, autopercepção da saúde e satisfação do paciente). A variável dependente deste estudo é intermediária, uma vez que as características pessoais da população influenciam o uso dos serviços odontológicos e estes resultam em desfechos de saúde que são a autopercepção da saúde e a satisfação do paciente com os serviços prestados ${ }^{5}$. Ressalta-se que a associação das questões socioeconômicas e subjetivas de saúde está relacionada ao uso, em estudos prévios ${ }^{6,13,14}$, de serviços odontológicos de forma regular. No entanto, numa ampla caracterização dos serviços odontológicos utilizados, considerando seus diferentes domínios e a avaliação da associação destes com o uso regular, não tem sido relatada na literatura, fato constatado no presente estudo.

A prevalência do uso regular de serviços odontológicos identificada neste estudo foi baixa. Ao considerar estudos com desenho metodológico similar ao presente inquérito, foram registradas prevalências semelhantes deste, com prevalência de uso entre 24,6\%, em Minas Gerais ${ }^{20}$, e de 32,8\%, no Rio Grande do Sul ${ }^{21}$.

O registro de maior prevalência de uso de forma regular entre pessoas com menor idade, 


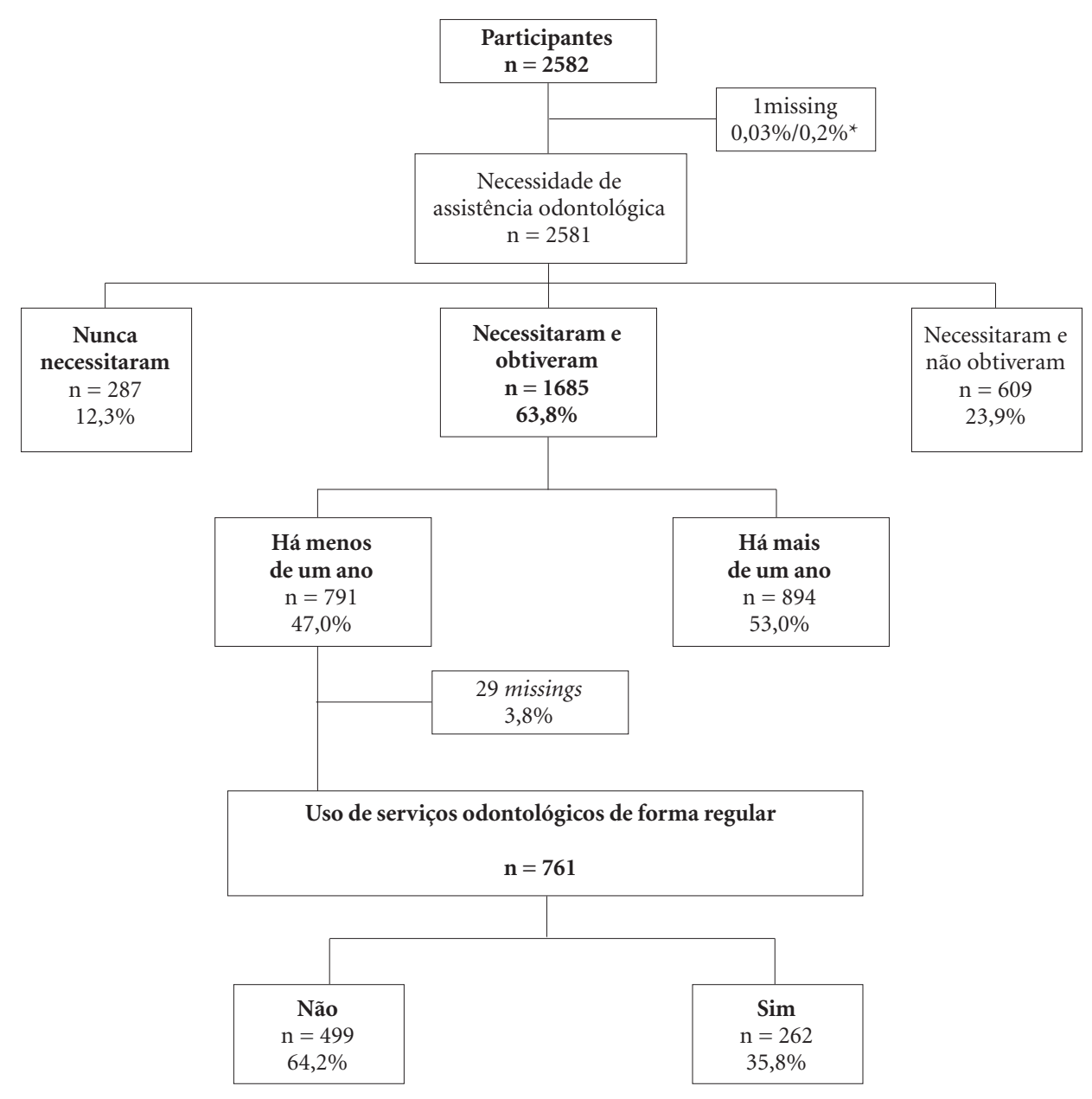

Figura 1. Fluxograma dos participantes do inquérito epidemiológico sobre uso da assistência odontológica, Montes Claros - MG, 2012.

identificado no presente estudo, corrobora com estudos internacionais e nacionais ${ }^{22-24}$. Entre finlandeses, pesquisa longitudinal identificou tendência de consulta de serviços odontológicos entre indivíduos jovens ${ }^{22}$. Estudos desenvolvidos em Passo Fundo (RS) ${ }^{23}$, Campinas $(\mathrm{SP})^{24}$ e em Ponta Grossa $(\mathrm{PR})^{25}$, também constataram tal tendência, sendo que, na pesquisa rio-grandense, o fato de possuir mais idade foi o que menos se associou à visita ao dentista, o que pode ter impactado pela maior prevalência de edentulismo entre pessoas nessa faixa etária ${ }^{23}$. Entretanto, estudo de base populacional dos fatores associados ao uso regular de serviços odontológicos em adultos de Bambuí (MG) identificou que a idade não estava associada à procura pelo serviço ${ }^{20}$.
Identificou-se maior chance de uso regular dos serviços odontológicos entre pessoas com maior nível de escolaridade. Sabe-se que a prevalência de uso regular de dentista por sujeitos com maior nível de escolaridade é cerca de 10 vezes maior do que entre aqueles menos escolarizados ${ }^{20}$. Tal comportamento pode ser explicado pela associação entre maior nível de escolaridade com melhor condição socioeconômica e melhor autopercepção da necessidade de cuidados odontológicos, sendo a autopercepção em saúde bucal um fator impactante na procura pelo serviço ${ }^{26,27}$. Estudos entre norte-americanos ${ }^{22}$, noruegueses ${ }^{9}$, canadenses $^{28}$ e brasileiros ${ }^{6,13,14,20,21,29,30}$ corroboram com o resultado constatado nesta pesquisa, uma vez que aqueles também registraram maior utili- 
Tabela 1. Características pessoais, sistema de atenção à saúde bucal, autopercepção da saúde bucal, saúde geral relatada, práticas pessoais e satisfação do paciente entre usuários dos serviços odontológicos de Montes Claros, MG, $2012(\mathrm{n}=761)$

\begin{tabular}{|c|c|c|}
\hline & $\mathbf{n}$ & $\%^{*}$ \\
\hline \multicolumn{3}{|l|}{ CARACTERÍSTICAS PESSOAIS } \\
\hline \multicolumn{3}{|l|}{ PREDISPOSIÇÃO } \\
\hline \multicolumn{3}{|l|}{ Sexo } \\
\hline Masculino & 211 & 27,2 \\
\hline Feminino & 550 & 72,8 \\
\hline \multicolumn{3}{|l|}{ Faixa etária em anos ${ }^{\star *}$} \\
\hline$>37 \leq 84$ & 350 & 48,2 \\
\hline$\geq 18 \leq 37$ & 400 & 51,8 \\
\hline \multicolumn{3}{|l|}{ Raça ou cor** } \\
\hline Parda/ preta & 558 & 72,1 \\
\hline Branca /amarela & 202 & 27,9 \\
\hline \multicolumn{3}{|l|}{ Escolaridade em anos ${ }^{* *}$} \\
\hline De 0 a 8 & 202 & 24,8 \\
\hline Igual ou maior que 9 & 559 & 75,2 \\
\hline \multicolumn{3}{|l|}{ Estado civil } \\
\hline Solteiro & 271 & 38,4 \\
\hline Separado/divorciado/viúvo & 76 & 9,8 \\
\hline Casado/ união estável & 414 & 51,8 \\
\hline \multicolumn{3}{|l|}{ DISPONIBILIDADE DE } \\
\hline \multicolumn{3}{|l|}{ RECURSOS } \\
\hline \multicolumn{3}{|l|}{ Renda per capita } \\
\hline Menor que um salário mínimo & 512 & 73,5 \\
\hline $\begin{array}{l}\text { Maior ou igual a um salário } \\
\text { mínimo }\end{array}$ & 171 & 26,5 \\
\hline \multicolumn{3}{|l|}{ Situação ocupacional ${ }^{\star *}$} \\
\hline Desempregado & 182 & 22,7 \\
\hline Nunca trabalhou & 88 & 13,3 \\
\hline Aposentado/pensionista & 60 & 8,9 \\
\hline Trabalha & 430 & 55,1 \\
\hline \multicolumn{3}{|l|}{$\begin{array}{l}\text { Meio de transporte utilizado para } \\
\text { chegar ao local da consulta }\end{array}$} \\
\hline Andou/bicicleta/outros & 302 & 43,1 \\
\hline Transporte público/ moto-táxi & 164 & 19,4 \\
\hline Veículo particular/táxi & 290 & 37,5 \\
\hline \multicolumn{3}{|l|}{$\begin{array}{l}\text { Tempo gasto para chegar ao local } \\
\text { da consulta }\end{array}$} \\
\hline$\geq 30 \leq 120$ minutos & 92 & 10,5 \\
\hline$\geq 15<30$ minutos & 247 & 30,5 \\
\hline$\geq 10<15$ minutos & 124 & 16,7 \\
\hline$\geq 0<10$ minutos & 294 & 42,3 \\
\hline \multicolumn{3}{|l|}{$\begin{array}{l}\text { SISTEMA DE ATENÇÃO À SAÚDE } \\
\text { BUCAL }\end{array}$} \\
\hline \multirow{2}{*}{\multicolumn{3}{|c|}{$\begin{array}{l}\text { O domicílio estava cadastrado no } \\
\text { Programa de Saúde da Família }\end{array}$}} \\
\hline & & \\
\hline Não & 252 & 39,7 \\
\hline Sim & 499 & 60,3 \\
\hline \multicolumn{3}{|l|}{ Local da consulta ${ }^{* *}$} \\
\hline Serviço público & 191 & 24,0 \\
\hline $\begin{array}{l}\text { Serviço particular/convênio } \\
\text { particular }\end{array}$ & 567 & 76,0 \\
\hline
\end{tabular}

\begin{tabular}{|c|c|c|}
\hline & $\mathbf{n}$ & $\%^{*}$ \\
\hline \multicolumn{3}{|l|}{$\begin{array}{l}\text { Prestador de assistência } \\
\text { odontológica }^{* *}\end{array}$} \\
\hline $\begin{array}{l}\text { Técnico de higiene/agente } \\
\text { comunitário/dentista prático/ } \\
\text { acadêmico }\end{array}$ & 50 & 5,9 \\
\hline Cirurgião dentista/clínico geral & 245 & 37,2 \\
\hline Cirurgião dentista/especialista & 380 & 57,0 \\
\hline \multicolumn{3}{|l|}{ Como o serviço foi pago ${ }^{\star \star}$} \\
\hline $\begin{array}{l}\text { Não pagou/ sistema público/ } \\
\text { filantrópico }\end{array}$ & 191 & 24,0 \\
\hline Plano de saúde & 129 & 16,7 \\
\hline Particular sem reembolso & 439 & 59,3 \\
\hline \multicolumn{3}{|l|}{$\begin{array}{l}\text { AUTOPERCEPÇÃO DA SAÚDE } \\
\text { BUCAL }\end{array}$} \\
\hline \multicolumn{3}{|l|}{$\begin{array}{l}\text { Como você classificaria a sua saúde } \\
\text { bucal?** }\end{array}$} \\
\hline Regular/ruim/péssima & 185 & 23,1 \\
\hline Ótima /boa & 572 & 76,9 \\
\hline \multicolumn{3}{|l|}{$\begin{array}{l}\text { Como você classificaria a sua } \\
\text { mastigação?**}\end{array}$} \\
\hline Regular/ruim/péssima & 264 & 32,0 \\
\hline Ótima /boa & 495 & 68,0 \\
\hline \multicolumn{3}{|l|}{$\begin{array}{l}\text { Como você classificaria a aparência } \\
\text { de seus dentes e gengivas?**}\end{array}$} \\
\hline Regular/ruim/péssima & 227 & 26,9 \\
\hline Ótima /boa & 533 & 73,1 \\
\hline \multicolumn{3}{|l|}{$\begin{array}{l}\text { Como você classificaria a sua fala } \\
\text { devido aos dentes e gengivas?** }\end{array}$} \\
\hline Regular/ruim/péssima & 105 & 12,4 \\
\hline Ótima /boa & 656 & 87,6 \\
\hline \multicolumn{3}{|l|}{$\begin{array}{l}\text { De que forma a sua saúde bucal } \\
\text { afeta o seu relacionamento com } \\
\text { outras pessoas?**}\end{array}$} \\
\hline $\begin{array}{l}\text { Mais ou menos/ muito/afeta } \\
\text { extremamente }\end{array}$ & 66 & 7,7 \\
\hline Não afeta/afeta pouco & 695 & 92,3 \\
\hline \multicolumn{3}{|l|}{$\begin{array}{l}\text { O quanto de dor os seus dentes e } \\
\text { gengivas lhe causaram nos últimos } \\
\text { seis meses?** }\end{array}$} \\
\hline Média/ muita /dor extrema & 139 & 15,6 \\
\hline Nenhuma/pouca dor & 622 & 84,4 \\
\hline \multicolumn{3}{|l|}{ SAÚDE GERAL RELATADA } \\
\hline \multicolumn{3}{|l|}{$\begin{array}{l}\text { Como você classificaria a sua saúde } \\
\text { geral?**}\end{array}$} \\
\hline Regular/ ruim/péssima & 188 & 23,4 \\
\hline Ótima/boa & 571 & 76,6 \\
\hline \multicolumn{3}{|l|}{ PRÁTICAS PESSOAIS } \\
\hline \multicolumn{3}{|l|}{ Já realizou o autoexame da boca?** } \\
\hline Não & 565 & 74,4 \\
\hline Sim & 193 & 25,6 \\
\hline
\end{tabular}


Tabela 1. continuação

\begin{tabular}{|c|c|c|c|c|c|}
\hline & n & $\%^{*}$ & & $\mathbf{n}$ & $\%^{*}$ \\
\hline SATISFAÇÃO DO PACIENTE & & & \multirow{3}{*}{$\begin{array}{l}\text {... Ter tempo suficiente para fazer } \\
\text { perguntas sobre seu problema } \\
\text { bucal ou tratamento?** }\end{array}$} & & \\
\hline Satisfação & & & & & \\
\hline \multirow{3}{*}{$\begin{array}{l}\text { Nem satisfeito, nem insatisfeito/ } \\
\text { parcialmente insatisfeito /muito } \\
\text { insatisfeito }\end{array}$} & \multirow[t]{3}{*}{69} & \multirow[t]{3}{*}{7,9} & & & \\
\hline & & & Regular/ruim/péssimo & 80 & 10,2 \\
\hline & & & Ótimo/bom & 669 & 89,8 \\
\hline $\begin{array}{l}\text { Muito satisfeito/parcialmente } \\
\text { satisfeito }\end{array}$ & 692 & 92,1 & $\begin{array}{l}\text {... A experiência de ter } \\
\text { informações sobre outros tipos de }\end{array}$ & & \\
\hline O prestador de assistência & & & exames ou tratamentos? ${ }^{* *}$ & & \\
\hline odontológica possui habilidades & & & Regular/ruim/péssimo & 121 & 15,2 \\
\hline adequadas? $^{* *}$ & & & Ótimo/bom & 630 & 84,8 \\
\hline Não & 42 & 5,7 & \multirow{2}{*}{$\begin{array}{l}\text {... Recebeu informações sobre } \\
\text { como evitar problemas bucais?** }\end{array}$} & & \\
\hline Sim & 712 & 94,3 & & & \\
\hline \multirow{3}{*}{$\begin{array}{l}\text { Materiais e equipamentos } \\
\text { do prestador de assistência } \\
\text { odontológica adequados para o } \\
\text { tratamento?** }\end{array}$} & & & \multirow{3}{*}{$\begin{array}{l}\text { Não } \\
\text { Sim } \\
\text {... Recebeu informações sobre } \\
\text { higiene bucal?** }\end{array}$} & 174 & 21,1 \\
\hline & & & & 584 & 78,9 \\
\hline & & & & & \\
\hline Não & 27 & 3,5 & \multirow{2}{*}{$\begin{array}{l}\text { Ocasionalmente/raramente/ } \\
\text { nunca }\end{array}$} & \multirow[t]{2}{*}{147} & \multirow[t]{2}{*}{19,2} \\
\hline Sim & 716 & 96,5 & & & \\
\hline \multirow{2}{*}{$\begin{array}{l}\text { QRSAO ....para sua última consulta } \\
\text { como você classificaria..(adaptado para } \\
\text { serviços odontológicos) }\end{array}$} & & & Sempre/frequentemente & 611 & 80,8 \\
\hline & & & $\begin{array}{l}\text {... Recebeu informações sobre } \\
\text { dieta/alimentação?** }\end{array}$ & & \\
\hline $\begin{array}{l}\text { DOMÍNIO PRONTIDÃO NO } \\
\text { ATENDIMENTO }\end{array}$ & & & $\begin{array}{l}\text { Ocasionalmente/raramente/ } \\
\text { nunca }\end{array}$ & 456 & 60,3 \\
\hline ... O tempo de viagem até & & & Sempre/frequentemente & 301 & 39,7 \\
\hline $\begin{array}{l}\text { o prestador de assistência } \\
\text { odontológica?**}\end{array}$ & & & $\begin{array}{l}\text {... Recebeu informações sobre } \\
\text { como evitar câncer de boca?**}\end{array}$ & & \\
\hline Regular/ruim/péssimo & 166 & 18,5 & Ocasionalmente/raramente/ & 516 & 70,0 \\
\hline Ótimo/bom & 589 & 81,5 & nunca & & \\
\hline $\begin{array}{l}\text {... O tempo que você esperou } \\
\text { antes de ser atendido?** }\end{array}$ & & & $\begin{array}{l}\text { Sempre/frequentemente } \\
\text {... Recebeu informações sobre }\end{array}$ & 242 & 30,0 \\
\hline Regular/ruim/péssimo & 146 & 18,9 & como realizar o autoexame da & & \\
\hline Ótimo/bom & 608 & 81,1 & boca?** & & \\
\hline $\begin{array}{l}\text { DOMÍNIO DIGNIDADE } \\
\text {... A experiência de ter sido }\end{array}$ & & & $\begin{array}{l}\text { Ocasionalmente/raramente/ } \\
\text { nunca }\end{array}$ & 594 & 79,3 \\
\hline recebido e tratado com & & & Sempre/frequentemente & 165 & 20,7 \\
\hline respeito?** & & & DOMÍNO AUTONOMIA & & \\
\hline Regular/ruim/péssimo & 23 & 3,1 & ... A experiência de estar & & \\
\hline Ótimo/bom & 728 & 96,9 & envolvido em tomar decisões & & \\
\hline DOMÍNIO PRIVACIDADE & & & sobre sua assistência ou & & \\
\hline ... Sua privacidade foi respeitada & & & tratamento $?^{* *}$ & & \\
\hline durante exames e tratamentos?** & & & Regular/ruim/péssimo & 81 & 11,6 \\
\hline Regular/ruim/péssimo & 16 & 2,1 & Ótimo/bom & 668 & 88,4 \\
\hline Ótimo/bom & 734 & 97,9 & DOMÍNIO ESCOLHA & & \\
\hline ... A maneira que o serviço foi & & & ... A liberdade que teve para & & \\
\hline prestado assegurou que você & & & escolher o seu prestador de & & \\
\hline poderia falar reservadamente ${ }^{\star *}$ & & & assistência odontológica?** & & \\
\hline Regular/ruim/péssimo & 58 & 7,1 & Regular/ruim/péssimo & 95 & 12,3 \\
\hline Ótimo/bom & 690 & 92,9 & Ótimo/bom & 652 & 87,7 \\
\hline DOMİNIO COMUNICAÇÃO & & & Procurou ir ao mesmo dentista ao & & \\
\hline ... A clareza com que o prestador & & & longo da vida?** & & \\
\hline explicou as coisas para você? ${ }^{* \star}$ & & & Não & 461 & 60,2 \\
\hline Regular/ruim/péssimo & 48 & 5,5 & Sim & 287 & 39,8 \\
\hline Ótimo/bom & 701 & 94,5 & & & ntinua \\
\hline
\end{tabular}


Tabela 1. continuação

\begin{tabular}{lrr}
\hline & n & $\%^{*}$ \\
\hline DOMÍNIO INFRAESTRUTURA & & \\
... A limpeza das salas dentro & & \\
do local de exame, incluindo os & & \\
banheiros?** & 29 & 3,3 \\
$\quad$ Regular/ruim/péssimo & 726 & 96,7 \\
$\quad$ Ótimo/bom & & \\
... A quantidade de espaço na & & \\
sala de espera e na sala onde foi & & \\
examinado?** & & \\
$\quad$ Regular/ruim/péssimo & 101 & 12,0 \\
$\quad$ Ótimo/bom & 653 & 88,0 \\
\hline
\end{tabular}

* Correção pelo efeito desenho. ${ }^{* *} \mathrm{O} \mathrm{n}^{\circ}$ de respondentes foi inferior ao $\mathrm{n}^{\circ}$ de participantes. ${ }^{* *}$ Salário Mínimo vigente $=$ $\mathrm{R} \$ 622,00(\$ 1,00=\mathrm{R} \$ 2,01$ maio 2012).

zação de serviços entre pessoas com maior nível de escolaridade. Tal informação norteia para a necessidade de uma avaliação acerca das desigualdades em saúde relacionadas às desigualdades socioeconômicas, principalmente no Brasil, país com diferenças regionais e culturais relevantes $^{30}$. Nessa perspectiva, é necessário avaliar os resultados da implantação de políticas de saúde, como a Política Nacional "Brasil Sorridente", que busca melhorar a condição de saúde e superar as desigualdades sociodemográficas na saúde bucal, considerando o acesso à assistência odontológica a todas as faixas etárias e níveis de atenção ${ }^{31}$.

Pessoas que classificaram a saúde bucal, a saúde geral e a aparência dos seus dentes e gengivas como "regular/ruim/péssima" tiveram menor chance de usar de forma regular os serviços odontológicos. Também foi encontrada associação entre o uso e relato de dor dos usuários nos últimos seis meses como média, muita dor ou dor extrema. Estudos prévios envolvendo idosos do Brasil encontraram associação entre uso, percepção da aparência dos dentes e gengivas como regular/ruim/péssima e relato de dor ${ }^{6,27,32}$. O modelo teórico utilizado neste estudo explica que as características pessoais da população influenciam os comportamentos de saúde, dentre eles o uso de serviços odontológicos, que por sua vez influenciam a autopercepção da saúde. Há que se ressaltar a característica de retroalimentação deste processo $^{10}$.

O fato de o prestador de assistência odontológica não possuir habilidades adequadas, na percepção do usuário, esteve associado ao menor uso dos serviços odontológicos de forma regular. A atuação na área da saúde é permeada pelas incertezas decorrentes da flutuação das demandas, falta de normatização de procedimentos e até mesmo condução inadequada do profissional. Nesse sentido, é importante a formação de profissionais com habilidades e competências adequadas ao setor de saúde do país, ressaltando-se que o plano de curso prevê essas habilidades. Nesse sentido, deve-se enfatizar esta formação e sempre que possível aproximar os alunos da realidade, de modo que, estes, ao ingressarem no mercado de trabalho, estejam mais preparados para as adversidades da rotina na odontologia. A falta de habilidade do profissional, na perspectiva do usuário, pode ser decorrente da condução inadequada do tratamento, da não resolução do problema, da falta de esclarecimentos ao paciente, da insegurança do profissional, ou até mesmo de descaso com o serviço, o que pode acarretar na influência dos comportamentos de saúde dos usuários ${ }^{33}$. O sistema de atenção à saúde bucal, é uma variável preditora importante nos resultados de saúde bucal, e tem como função proporcionar ao uso formas dos serviços de saúde odontológica, tanto nos níveis: preventivo, curativo ou restaurador ${ }^{10}$.

Maior chance de uso de forma regular no último ano também foi encontrada entre pessoas que classificaram como sempre/frequentemente o fato de receber informações sobre higiene bucal e sobre dieta e alimentação. E menor chance foi verificada em indivíduos que classificaram como sempre/frequentemente de terem tempo suficiente para fazerem perguntas sobre o problema bucal ou tratamento. Os achados demonstram a importância das atitudes dos profissionais da área da saúde, tais como: cumprimentar o paciente, ser atencioso, interessar-se pelo caso, utilizar linguagem clara, explicar o problema e dar ao paciente oportunidade para esclarecer suas dúvidas. Essas atitudes podem gerar maior satisfação e consequente uso dos serviços de forma ideal ${ }^{29}$. O sistema de atenção à saúde bucal, sobretudo a prática dos profissionais, promove bons comportamentos de saúde bucal que influenciam tanto no uso formal dos serviços, como na avaliação satisfatória da assistência prestada ${ }^{10,34}$. Vale ressaltar que o conhecimento de saúde bucal pode ter relação com os determinantes sociais, haja visto que mulheres com melhor nível socioeconômico conhecem mais adequadamente os cuidados para com a saúde bucal dos seus filhos, bem como são mais frequentemente orientadas sobre tais cuidados ${ }^{26}$. 
Tabela 2. Análise bivariada entre o uso de serviços odontológicos de forma regular e características pessoais, sistema de atenção á saúde bucal, autopercepção da saúde bucal, saúde geral relatada, práticas pessoais e satisfação do paciente entre usuários dos serviços odontológicos de Montes Claros, MG, 2012, (n=761).

\begin{tabular}{|c|c|c|c|c|c|}
\hline \multirow[b]{2}{*}{ CARACTERÍSTICAS PESSOAIS } & \multicolumn{2}{|c|}{$\begin{array}{l}\text { Uso de serviços } \\
\text { odontológicos } \\
\text { de forma regular }\end{array}$} & \multirow[t]{2}{*}{$\mathrm{OR}^{* *}$} & \multirow[t]{2}{*}{$\mathrm{IC}_{95 \%}^{* *}$} & \multirow[t]{2}{*}{$\mathbf{p}$} \\
\hline & Não & Sim & & & \\
\hline PREDISPOSIÇÃO & $\%^{* *}$ & $\%^{* *}$ & & & \\
\hline \multicolumn{6}{|l|}{ Sexo } \\
\hline Masculino & 54,4 & 45,6 & 1,00 & & \\
\hline Feminino & 67,8 & 32,2 & 0,57 & $0,38-0,86$ & 0,010 \\
\hline \multicolumn{6}{|l|}{ Faixa etária em anos** } \\
\hline$>37 \leq 84$ & 7408 & 25,2 & 1,00 & & \\
\hline$\geq 18 \leq 37$ & 55,5 & 44,5 & 2,37 & $1,55-3,65$ & 0,000 \\
\hline \multicolumn{6}{|l|}{ Raça ou $\operatorname{cor}^{* *}$} \\
\hline Parda/ preta & 64,5 & 35,5 & 1,00 & & \\
\hline Branca /amarela & 63,3 & 36,7 & 1,05 & $0,69-1,61$ & 0,813 \\
\hline \multicolumn{6}{|l|}{ Escolaridade em anos ${ }^{\star *}$} \\
\hline De 0 a 8 & 87,1 & 12,9 & 1,00 & & \\
\hline Igual ou maior que 9 & 56,6 & 43,4 & 5,21 & $3,21-8,43$ & 0,000 \\
\hline \multicolumn{6}{|l|}{ Estado civil } \\
\hline Solteiro & 54,0 & 46,0 & 1,00 & & \\
\hline Separado/divorciado/viúvo & 84,1 & 15,9 & 0,22 & $0,09-0,52$ & 0,003 \\
\hline Casado/ união estável & 67,9 & 32,1 & 0,55 & $0,38-0,80$ & 0,045 \\
\hline \multicolumn{6}{|l|}{ DISPONIBILIDADE DE RECURSOS } \\
\hline \multicolumn{6}{|l|}{ Renda per capita } \\
\hline Menor que um salário mínimo & 72,2 & 27,8 & 1,00 & & \\
\hline Maior ou igual a um salário mínimo & 50,0 & 50,0 & 2,60 & $1,72-3,93$ & 0,000 \\
\hline \multicolumn{6}{|l|}{ Situação ocupacional ${ }^{\star *}$} \\
\hline Desempregado & 68,4 & 31,6 & 1,00 & & \\
\hline Nunca trabalhou & 51,7 & 48,3 & 2,01 & $1,11-3,67$ & 0,389 \\
\hline Aposentado/pensionista & 73,2 & 26,8 & 0,79 & $0,40-1,57$ & 0,030 \\
\hline Trabalha & 63,9 & 36,1 & 1,22 & $0,76-1,97$ & 0,099 \\
\hline \multicolumn{6}{|c|}{$\begin{array}{l}\text { Meio de transporte utilizado para chegar ao local da } \\
\text { consulta }\end{array}$} \\
\hline Andou/bicicleta/outros & 70,1 & 29,9 & 1,00 & & \\
\hline Transporte público/ moto-táxi & 69,2 & 30,8 & 1,04 & $0,58-1,88$ & 0,007 \\
\hline Veículo particular/táxi & 54,3 & 45,7 & 1,97 & $1,22-3,19$ & 0,008 \\
\hline \multicolumn{6}{|c|}{ Tempo gasto para chegar ao local da consulta ${ }^{* *}$} \\
\hline$\geq 0<10$ minutos & 63,8 & 36,2 & 1,00 & & \\
\hline$\geq 10<15$ minutos & 60,0 & 40,0 & 1,18 & $0,69-1,99$ & 0,531 \\
\hline$\geq 15<30$ minutos & 66,2 & 33,8 & 0,90 & $0,57-1,43$ & 0,645 \\
\hline$\geq 30 \leq 120$ minutos & 64,7 & 35,3 & 0,96 & $0,46-2,03$ & 0,919 \\
\hline \multicolumn{6}{|l|}{ SISTEMA DE ATENÇÃO À SAÚDE BUCAL } \\
\hline \multicolumn{6}{|c|}{$\begin{array}{l}\text { O domicílio estava cadastrado no Programa de Saúde } \\
\text { da Família** }\end{array}$} \\
\hline Não & 53,8 & 46,2 & 1,00 & & \\
\hline Sim & 71,0 & 29,0 & 0,48 & $0,29-0,77$ & 0,004 \\
\hline \multicolumn{6}{|l|}{ Local da consulta ${ }^{* *}$} \\
\hline Serviço público & 88,1 & 11,9 & 1,00 & & \\
\hline Serviço particular/convênio particular & 57,0 & 43,0 & 5,56 & $2,94-10,54$ & 0,000 \\
\hline
\end{tabular}


Tabela 2. continuação

\begin{tabular}{|c|c|c|c|c|c|}
\hline \multirow{2}{*}{ Prestador de assistência odontológica ${ }^{\star \star}$} & \multicolumn{2}{|c|}{$\begin{array}{l}\text { Uso de serviços } \\
\text { odontológicos } \\
\text { de forma regular }\end{array}$} & \multirow[t]{2}{*}{$\mathrm{OR}^{* *}$} & \multirow[t]{2}{*}{$\mathrm{IC}_{95 \%}^{* *}$} & \multirow[t]{2}{*}{$\mathbf{p}$} \\
\hline & & & & & \\
\hline $\begin{array}{l}\text { Técnico de higiene/agente comunitário/dentista } \\
\text { prático/acadêmico }\end{array}$ & 76,1 & 23,9 & 1,00 & & \\
\hline Cirurgião dentista/clínico geral & 73,2 & 26,8 & 1,16 & $0,43-3,15$ & 0,067 \\
\hline Cirurgião dentista/especialista & 56,0 & 44,0 & 2,50 & $0,93-6,68$ & 0,000 \\
\hline \multicolumn{6}{|l|}{ Como o serviço foi pago ${ }^{\star *}$} \\
\hline Não pagou/ sistema público/filantrópico & 88,1 & 11,9 & 1,00 & & \\
\hline Plano de saúde & 44,0 & 56,0 & 9,41 & $4,48-19,58$ & 0,006 \\
\hline Particular sem reembolso & 59,9 & 40,1 & 4,94 & $2,64-9,22$ & 0,000 \\
\hline \multicolumn{6}{|l|}{ AUTOPERCEPÇÃO DA SAÚDE BUCAL } \\
\hline \multicolumn{6}{|l|}{ Como você classificaria a sua saúde bucal? l* $^{* *}$} \\
\hline Regular/ruim/péssima & 91,9 & 8,1 & 1,00 & & \\
\hline Ótima /boa & 56,0 & 44,0 & 8,88 & $3,91-20,16$ & 0,000 \\
\hline \multicolumn{6}{|l|}{ Como você classificaria a sua mastigação?** } \\
\hline Regular/ruim/péssima & 74,8 & 25,2 & 1,00 & & \\
\hline Ótima /boa & 59,0 & 41,0 & 2,07 & $1,25-3,42$ & 0,007 \\
\hline \multicolumn{6}{|l|}{$\begin{array}{l}\text { Como você classificaria a aparência de seus dentes e } \\
\text { gengivas?** }\end{array}$} \\
\hline Regular/ruim/péssima & 82,3 & 17,7 & 1,00 & & \\
\hline Ótima /boa & 57,4 & 42,6 & 3,46 & $2,07-5,79$ & 0,000 \\
\hline \multicolumn{6}{|l|}{$\begin{array}{l}\text { Como você classificaria a sua fala devido aos dentes e } \\
\text { gengivas?**}\end{array}$} \\
\hline Regular/ruim/péssima & 84,7 & 15,3 & 1,00 & & \\
\hline Ótima /boa & 61,3 & 38,7 & 3,49 & $1,72-7,10$ & 0,001 \\
\hline \multicolumn{6}{|l|}{$\begin{array}{l}\text { De que forma a sua saúde bucal afeta o seu } \\
\text { relacionamento com outras pessoas?** }\end{array}$} \\
\hline Mais ou menos/muito/afeta extremamente & 81,5 & 18,5 & 1,00 & & \\
\hline Não afeta/afeta pouco & 62,7 & 37,3 & 2,62 & $0,92-7,48$ & 0,069 \\
\hline \multicolumn{6}{|l|}{$\begin{array}{l}\text { O quanto de dor os seus dentes e gengivas lhe } \\
\text { causaram nos últimos seis meses?**}\end{array}$} \\
\hline Média/ muita /dor extrema & 85,9 & 14,1 & 1,00 & & \\
\hline Nenhuma/pouca dor & 60,1 & 39,9 & 4,05 & $2,05-8,03$ & 0,000 \\
\hline \multicolumn{6}{|l|}{ SAÚDE GERAL RELATADA } \\
\hline \multicolumn{6}{|l|}{ Como você classificaria a sua saúde geral?** } \\
\hline Regular/ ruim/péssima & 84,2 & 15,8 & 1,00 & & \\
\hline Ótima/boa & 57,9 & 42,1 & 3,86 & $2,47-6,04$ & 0,000 \\
\hline \multicolumn{6}{|l|}{ PRÁTICAS PESSOAIS } \\
\hline \multicolumn{6}{|l|}{ Já realizou o autoexame da boca?** } \\
\hline Não & 66,2 & 33,8 & 1,00 & & \\
\hline Sim & 58,6 & 41,4 & 1,49 & $1,10-2,01$ & 0,130 \\
\hline \multicolumn{6}{|l|}{ SATISFAÇÃO DO PACIENTE } \\
\hline \multicolumn{6}{|l|}{ Satisfação } \\
\hline $\begin{array}{l}\text { Nem satisfeito, nem insatisfeito/parcialmente } \\
\text { insatisfeito /muito insatisfeito }\end{array}$ & 93,4 & 6,6 & 1,00 & & \\
\hline Muito satisfeito/parcialmente satisfeito & 61,6 & 38,4 & 8,79 & $2,69-28,67$ & 0,001 \\
\hline
\end{tabular}


Tabela 2. continuação

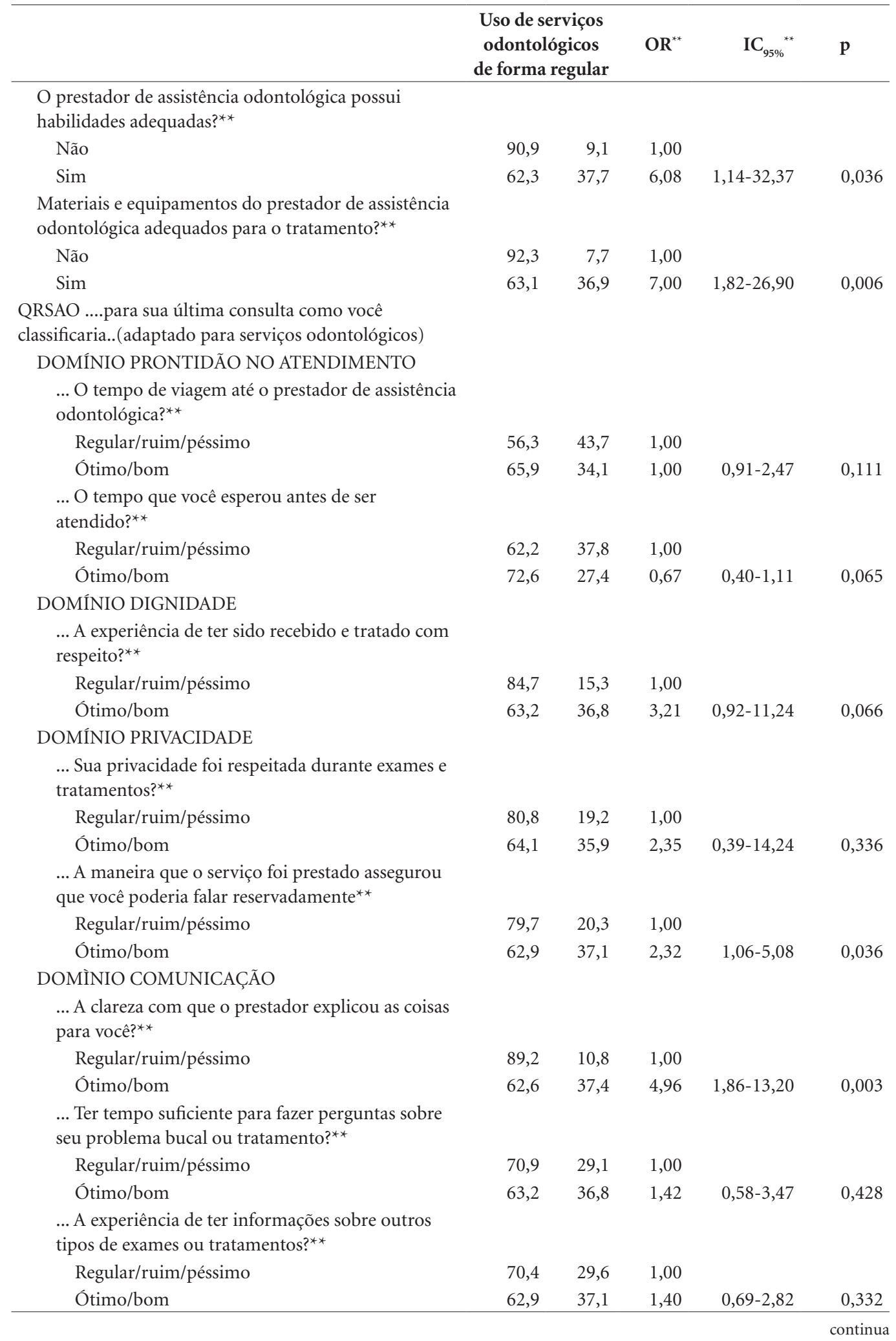


Tabela 2. continuação

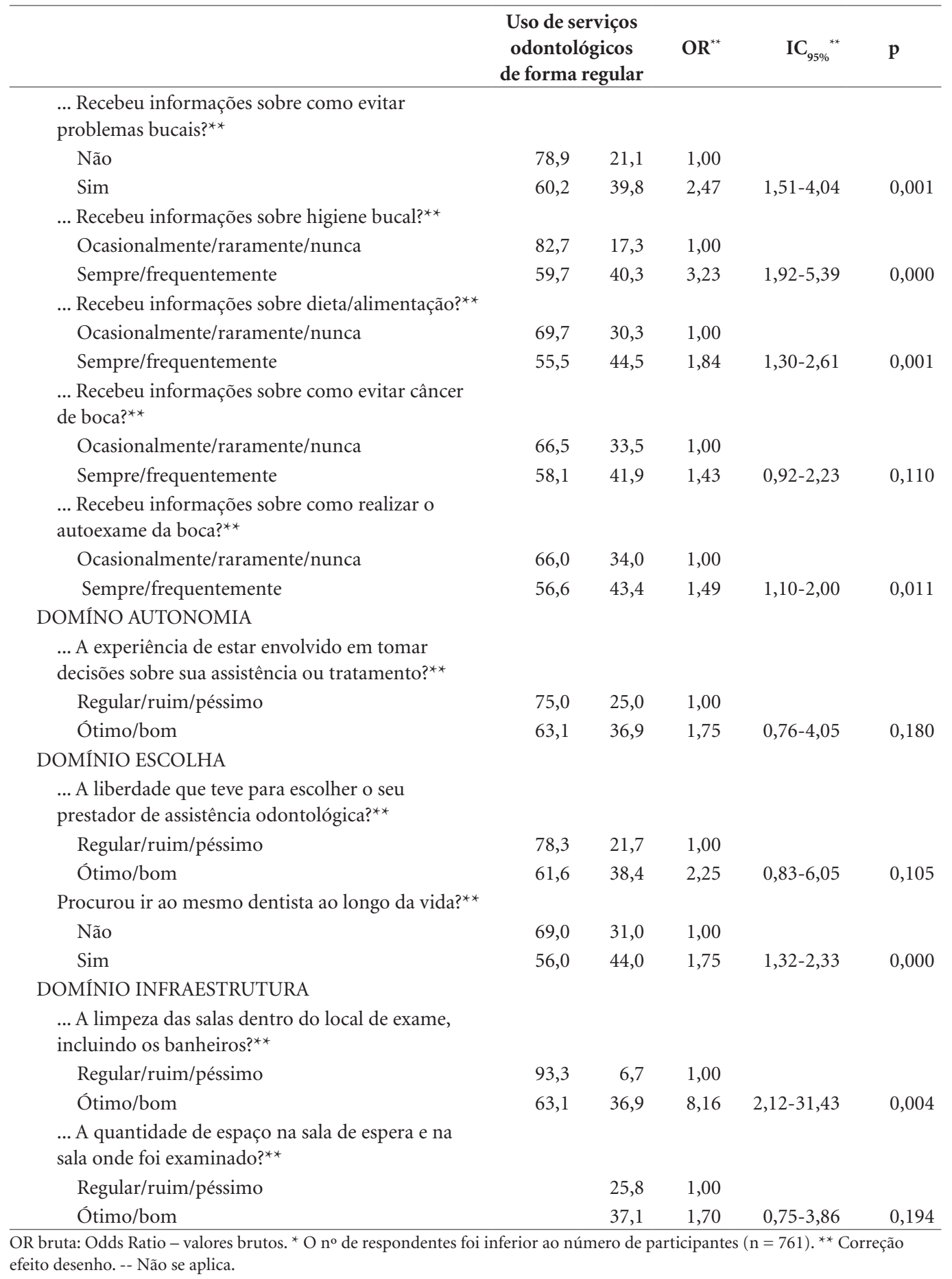

No que diz respeito às questões relacionadas ao modelo teórico de Andersen e Davidson, identificou-se associação das variáveis presentes nos grupos propostos e o uso de forma regular dos serviços no último ano. Este é um estudo de base populacional, representativo da população adul- ta da cidade de Montes Claros e que, ao estudar a utilização dos serviços, incluiu indivíduos que o usaram e que não o usaram, o que proporciona critério de comparação. Entretanto, há que se ressaltar que o uso de serviços odontológicos de forma regular é um comportamento socialmente 
Tabela 3. Análise múltipla entre o uso de serviços odontológicos de forma regular e características pessoais, sistema de atenção á saúde bucal, autopercepção da saúde bucal, saúde geral relatada, práticas pessoais e satisfação do paciente entre usuários dos serviços odontológicos de Montes Claros, MG, 2012, (n = 761).

\begin{tabular}{|c|c|c|c|}
\hline & $\mathrm{OR}_{\text {ajustada }}^{*}$ & $\mathrm{IC}_{95 \%}^{*}$ & $\mathrm{P}$ \\
\hline \multicolumn{4}{|l|}{ CARACTERÍSTICAS PESSOAIS } \\
\hline \multicolumn{4}{|l|}{ PREDISPOSIÇÃO } \\
\hline \multicolumn{4}{|l|}{ Faixa etária em anos** } \\
\hline$>37 \leq 84$ & 1,00 & & \\
\hline$\geq 18 \leq 37$ & 1,82 & $1,14-2,91$ & 0,050 \\
\hline \multicolumn{4}{|l|}{ Escolaridade em anos ${ }^{\star *}$} \\
\hline De 0 a 8 & 1,00 & & \\
\hline Igual ou maior que 9 & 3,76 & $2,14-6,60$ & 0,000 \\
\hline \multicolumn{4}{|l|}{ AUTOPERCEPÇÃO DA SAÚDE BUCAL } \\
\hline \multicolumn{4}{|l|}{ Como você classificaria a sua saúde bucal?** } \\
\hline Regular/ruim/péssima & 1,00 & & \\
\hline Ótima /boa & 3,87 & $2,03-7,37$ & 0,000 \\
\hline \multicolumn{4}{|c|}{ Como você classificaria a aparência de seus dentes e gengivas? ${ }^{2 *}$} \\
\hline Regular/ruim/péssima & 1,00 & & \\
\hline Ótima /boa & 1,90 & $1,06-3,38$ & 0,037 \\
\hline \multicolumn{4}{|c|}{$\begin{array}{l}\text { O quanto de dor os seus dentes e gengivas lhe causaram nos últimos } \\
\text { seis meses?** }\end{array}$} \\
\hline Média/ muita /dor extrema & 1,00 & & \\
\hline Nenhuma/pouca dor & 3,00 & $1,47-6,14$ & 0,004 \\
\hline \multicolumn{4}{|l|}{ SAÚDE GERAL RELATADA } \\
\hline \multicolumn{4}{|l|}{ Como você classificaria a sua saúde geral?** } \\
\hline Regular/ruim/péssima & 1,00 & & \\
\hline Ótima /boa & 1,81 & $1,06-3,09$ & 0,034 \\
\hline \multicolumn{4}{|l|}{ SATISFAÇÃO DO PACIENTE } \\
\hline \multicolumn{4}{|c|}{$\begin{array}{l}\text { O prestador de assistência odontológica possui habilidades } \\
\text { adequadas?** }\end{array}$} \\
\hline Não & 1,00 & & \\
\hline Sim & 4,19 & $0,93-18,96$ & 0,034 \\
\hline \multicolumn{4}{|l|}{ DOMİNIO COMUNICAÇÃO } \\
\hline \multicolumn{4}{|c|}{$\begin{array}{l}\text {... Ter tempo suficiente para fazer perguntas sobre seu problema } \\
\text { bucal ou tratamento?** }\end{array}$} \\
\hline Ótimo/bom & 1,00 & & \\
\hline Regular/ruim/péssimo & 0,40 & $0,16-0,96$ & 0,010 \\
\hline \multicolumn{4}{|c|}{... Recebeu informações sobre higiene bucal?** } \\
\hline Ocasionalmente/raramente/nunca & 1,00 & & \\
\hline Sempre/frequentemente & 2,08 & $1,26-3,43$ & 0,005 \\
\hline \multicolumn{4}{|c|}{... Recebeu informações sobre dieta/alimentação?** } \\
\hline Ocasionalmente/raramente/nunca & 1,00 & & \\
\hline Sempre/frequentemente & 1,66 & $1,07-2,57$ & 0,031 \\
\hline
\end{tabular}

* Correção efeito desenho. ${ }^{* *} \mathrm{O} n^{\circ}$ de respondentes foi inferior ao número de participantes $(\mathrm{n}=761)$.

desejável. Nesse sentido, pessoas podem superestimar as consultas realizadas e, dificilmente, esse comportamento poderá ser verificado em sentido contrário ${ }^{33}$, tornando-se uma limitação do estudo. Dentre outras limitações deste estudo, ressalta-se o fato de o processo de avaliação ser dinâmico, quanto ao uso de serviços odontoló- gicos na perspectiva e nas variáveis investigadas. Portanto, causas e efeitos certamente variam ao longo do tempo. Sendo este um estudo transversal, pode ocorrer viés de causalidade reversa e, sendo seccional, não permitir estabelecer a relação temporal entre as associações observadas, a interpretação dos achados ser limitada. Por outro 
lado, destaca-se o fato de se tratar de um estudo de base populacional, com uma amostra representativa do município e que aborda um tema ainda pouco explorado.

Por fim, os resultados encontrados devem ser considerados. Conclui-se que o uso de serviços odontológicos de forma regular foi relativamente baixo. Considerando-se a alta prevalência de problemas bucais de que a população brasileira é acometida e a importância do uso de serviços de saúde bucal de forma regular na prevenção desses problemas, tal prática deve ser incentivada. Constata-se ainda que o uso de forma regular

\section{Colaboradores}

DL Carreiro, RC Ferreira e AMEBL Martins participaram do delineamento, coleta e análise dos dados e redação do artigo. JGS Souza e WLM Coutinho participaram da análise de dados e redação do artigo. E Ferreira e Ferreira participou da revisão crítica. manteve-se associado a características pessoais dos indivíduos, condições subjetivas de saúde e característica do serviço odontológico utilizado. Essas associações demonstram a possível relação entre melhores condições socioeconômicas, melhor percepção da saúde e das condições do serviço odontológico e prestador da assistência, com um maior uso de forma regular. Tais achados devem ser considerados na melhoria das políticas públicas de saúde bucal que visem ao incentivo do uso de serviços odontológicos de forma regular como forma de prevenção a agravos bucais e monitoramento das condições de saúde bucal dos indivíduos.

\section{Agradecimentos}

À FAPEMIG pela bolsa de Raquel Conceição Ferreira. 


\section{Referências}

1. Pereira AC. Odontologia em saúde coletiva: planejando ações e promovendo saúde. Porto Alegre; Artmed; 2007.

2. Souza TMS, Roncalli AG. Saúde bucal no Programa Saúde da Família: uma avaliação do modelo assistencial. Cad Saude Publica 2007; 23(11):2727-2739.

3. Mattos GCM, Ferreira e Ferreira E, Leite ICG, Greco RM. A inclusão da equipe de saúde bucal na Estratégia Saúde da Família: entraves, avanços e desafios. Cien Saude Colet 2014; 19(2):373-382.

4. Moyses SJ, Pucca Junior GA, Paludetto Junior M, Moura L. Avanços e desafios à Política de Vigilância à Saúde Bucal no Brasil. Rev Saude Publica 2013; 47(Supl. 3):161-167.

5. Pinheiro RS, Torres TZG. Uso de serviços odontológicos entre os estados do Brasil. Cien Saude Colet 2006; 11(4):999-910.

6. Martins AMEBL, Haikal DS, Pereira SM, Barreto SM. Uso de serviços odontológicos por rotina entre idosos brasileiros: Projeto SB Brasil. Cad Saude Publica 2008; 24(7):1651-1666.

7. Ferreira CO, Antunes JLF, Andrade FB. Fatores associados à utilização dos serviços odontológicos por idosos brasileiros. Rev Saude Publica 2013; 47(Supl. 3):90-97.

8. Brasil. Ministério da Saúde (MS). Secretaria de Atenção à Saúde. Departamento de Atenção Básica. Coordenação Nacional de Saúde Bucal. Projeto SB Brasil 2010: Condições de Saúde Bucal da População Brasileira, Resultados Principais. Brasília: MS, 2011.

9. Swank ME, Vernon SW, Lairson DR. Patterns of preventive dental behavior. Public Health Reports 1986; 101(2)175-184.

10. Andersen RM, Davidson PL. Ethnicity, aging, and oral health outcomes: a conceptual framework. Adv Dent Res 1997; 11(2):203-209.

11. Schwarz E, Hansen ER. Utilization of dental services in the adult Danish population 1975. Community Dent Oral Epidemiol 1976; 4(6):221-226.

12. Reisine ST. Dental disease and work loss. J Dent Res 1984; 63(9):1158-1161.

13. Camargo MBJ, Barros AJD, Frazão P, Matijasevich A, Santos IS, Peres MA, Peres KG. Preditores da realização de consultas odontológicas de rotina e por problemas em pré-escolares. Rev Saude Publica 2012; 46(1):87-87.

14. Machado LP, Camargo MBJ, Jeronymo JCM, Bastos GAN. Uso regular de serviços odontológicos entre adultos idosos em região vulnerável no sul do Brasil. Rev Saude Publica 2012; 46(3):526-533.

15. Martins AMEBL, Barreto SM, Pordeus IA. Características associadas ao uso de serviços odontológicos entre idosos dentados e edentados no Sudeste do Brasil: Projeto SB Brasil. Cad Saúde Publica 2008; 24(1):81-92.

16. Rodrigues CAQ, Silva PLV, Caldeira AP, Pordeus IA, Ferreira RC, Martins AMEBL. Fatores associados à satisfação com serviços odontológicos entre idosos. Rev Saude Publica 2012; 46(6):1039-1050.

17. Brasil. Ministério da Educação (ME). LDB. Lei 9394/96 - Lei de Diretrizes e Bases da Educação Nacional. [documento da Internet]. [acessado 2014 Jan 24]. Disponível em: http:www.planalto.gov.br
18. Pereira JM, Salomão SR, Cinoto RW, Mendieta L, Sacai PY, Berezovsky A, Belfort Junior R. Avaliação dos serviços de assistência ocular em população urbana de baixa renda da cidade de São Paulo - Brasil. Arq Bras Oftalmol 2009; 72(3):332-340.

19. Martins AMEBL, Ferreira RC, Santos-Neto PE, Carreiro DL, Souza JGS, Ferreira e Ferreira E. Insatisfação dos usuários com a assistência odontológica: estudo domiciliar populacional. Rev Saúde Publica 2015; 49:51.

20. Matos DL, Lima-Costa MFF, Guerra HL, Marcenes W. Projeto Bambuí: estudo de base populacional dos fatores associados com o uso regular de serviços odontológicos em adultos. Cad Saude Publica 2001; 17(3):66616668.

21. Camargo MBJ, Dumith SC, Barros AJD. Uso regular de serviços odontológicos entre adultos: padrões de utilização e tipos de serviços. Cad Saude Publica 2009; 25(9):1894-1896.

22. Suominen-Taipale AL, Widstrom E, Alanen P, Uutela A. Trends in self-reported use of dental services among finish adults during two decades. Community Dent Health 2000; 17(1):31-37.

23. Mattos DA, Lehnen A, Trentin MS, Silva SO, Carli JP, Linden MSS. Perfil dos pacientes atendidos no Setor de Exames e Triagem da Faculdade de Odontologia da Universidade de Passo Fundo, Rio Grande do Sul. RGO 2009; 57(4):437-441.

24. Rocha JMY. Ocorrência de doenças sistêmicas, reações alérgicas e uso de medicamentos pelos pacientes do Centro Cirúrgico da Faculdade de Odontologia de Piracicaba (Unicamp) de 1997 a 2000. Rev Bras Cir Traum Buco-Maxilo-Facial 2004; 1(1):3-7.

25. Baldani MH, Antunes JLF. Inequalities in access and utilization of dental services: a cross-sectional study in an area covered by the Family Health Strategy. Cad Saude Publica 2011; 27(2):272-283.

26. Heft MW, Gilbert GH, Shelton BJ, Duncan RP. Relationship of dental status, sociodemographic status, and oral symptoms to perceived need for dental care. Community Dent Oral Epidemiol 2003; 31(5):351-360.

27. Martins AMEB, Barreto SM, Pordeus IA. Uso de serviços odontológicos entre idosos brasileiros. Rev Panam Salud Publica 2007; 22(5):308-315.

28. Locker D, Leake JL, Lee J, Main PA, Hics T, Hamiltom M. Utilization of dental services by older adults in four Ontario communities. J Can Dent Assoc 1991; 57:879886.

29. Araujo CS, Lima RC, Peres MA, Barros AJD. Utilização de serviços odontológicos e fatores associados: um estudo de base populacional no Sul do Brasil. Cad Saude Publica 2009; 25(5):1063-1072.

30. Barros AJD, Bertoldi AD. Desigualdades na utilização e no acesso a serviços odontológicos: uma avaliação em nível nacional. Cien Saude Colet 2002; 7(4):709-717.

31. Brasil. Ministério da Saúde (MS). Diretrizes da Política Nacional de Saúde Bucal. [documento da Internet] 2004 [acessado 2014 Maio 20]. Disponível em: www. saude.gov.br/bucal. 
32. Vilela EA, Martins AMEBL, Barreto SM, Vargas AMD, Ferreira RC. Association between self-rated oral appearance and the need for dental prostheses among elderly Brazilians. Braz Oral Res 2013; 27(3):203-210.

33. Amâncio Filho A. Dilemas e desafios da formação profissional em saúde. Interface (Botucatu) 2004; 8(15): 375-380.

34. Martins AMEBL, Jardim LA, Souza JGS, Rodrigues CAQ, Ferreira RC, Pordeus IA. Is the negative evaluation of dental services among the Brazilian elderly population associated with the type of service? Rev Bras Epidemiol 2014; 17(1):71-90.

Artigo apresentado em 11/06/2015

Aprovado em 07/05/2016

Versão final apresentada em 09/05/2016 